\title{
Low light and over exposed Image Enhancement using Weight Matrix Technique
}

\author{
Premanand Ghadekar \\ VIT Pune
}

\author{
Rohit Kale \\ VIT Pune
}

\author{
Nikhil Agrawal \\ VIT Pune
}

\author{
Atharva Pophale \\ VIT Pune
}

\author{
Saurabh Rudrawar \\ VIT Pune
}

\begin{abstract}
Due to the low exposure, the low light pictures aren't so conductive to human observation and computer vision algorithm. Though many image improvement techniques are planned to unravel this drawback, existing ways ultimately introduce under and over improvement of contrast. This paper recommends an image contrast algorithm to produce a certain improvement of contrast. Specifically, the weight matrix for image fusion using illumination estimation techniques is designed. Then algorithm tend to introduce camera response model to synthesize multi-exposure pictures. Next, algorithm tend to find the most effective exposure ratio so the artificial image is well-exposed within the regions wherever the first image under-exposed. Finally, the input image and the artificial image are fused according to the weight matrix to get the improvement result. Experiments show that this methodology gets results with less contrast and lightness distortion compared to that of many state- of-the-art methods. The proposed algorithm also preserves the information content by reducing the overexposure and gives the best results in terms of processing time.
\end{abstract}

\section{Keywords}

Image enhancement, contrast enhancement, exposure comparison, exposure, fusion, weight matrix

\section{INTRODUCTION}

Image improvement techniques are widely utilized in image processing. In general, it builds the input pictures look better and be more appropriate for suitable algorithms. contrast improvement, as one kind of improvement techniques, and reveal the knowledge of the under-exposed regions in a picture. many contrast improvement techniques are proposed including histogram-based, Retinex-based and dehaze-based methods. A color image may be pictured as a threedimensional array [1]. the only scheme of contrast improvement performs the same processing for every component. for instance, the earliest image improvement strategies use a non-linear monotonic operate (power-law, logarithm, etc.) for gray-level mapping. Considering the uneven distribution of parts in parts grey levels, histogram equalization (HE) is wide used for improving contrast. several extensions to $\mathrm{HE}$ is planned to require some restrictions under consideration like brightness preservation and distinction limitation [2].

However, HE-based strategies forever from overenhancement and cause unreasonable results. Imitating the human sensory system [3], Retinex theory is additionally wide employed in image improvement. By separating reflectance from the illumination, Retinex-based algorithms enhances the main points clearly. However, these strategies always from halo- like whole thing within the high distinction region. In recent years, some strategies borrowed the de-haze technique to distinction improvement, and wins \} good subjective visual eff ect [5]. however, this work might cause color distortion as a result of contrast over-enhancement. Although image contrast improvement strategies are studied for many years, the definition of a decent improvement result's still not welldefined [6]. Additionally, no reference is offer for existing low-light improvement rule to find the over- and underenhancement region. The system has a tendency to notice that the photographs that only solely in exposures may be used as a reference for improvement algorithms. Because the exposure increases, some under- exposed regions become well-exposed. The improvement result ought to keep the wellexposed regions unchanged and enhance the under-exposed region [7]. Meanwhile, the distinction of increased regions ought to be according to the reference image that correct exposes the region.

In this paper, algorithm have a tendency to planned a replacement framework to assist mitigate under- and overenhancement drawback. The framework is predicated on exposure fusion among multi-exposure images synthesized from the input image by the camera response model. Based on the framework, the enhancement algorithm is proposed that gets results with less contrast and lightness distortion compared to many state-of-the-art strategies.

For the enhancement of over exposure images, very few techniques are available and these techniques does not generate the quality results for the highly over exposed images. For over exposure, system is again determining the value of exposure ratio $\mathrm{k}$, if the value of $\mathrm{k}$ is greater than the threshold decided then system labels that area as over exposure area and again determines the new value of $\mathrm{k}$.

\section{PROPOSED FLOW DIAGRAM}




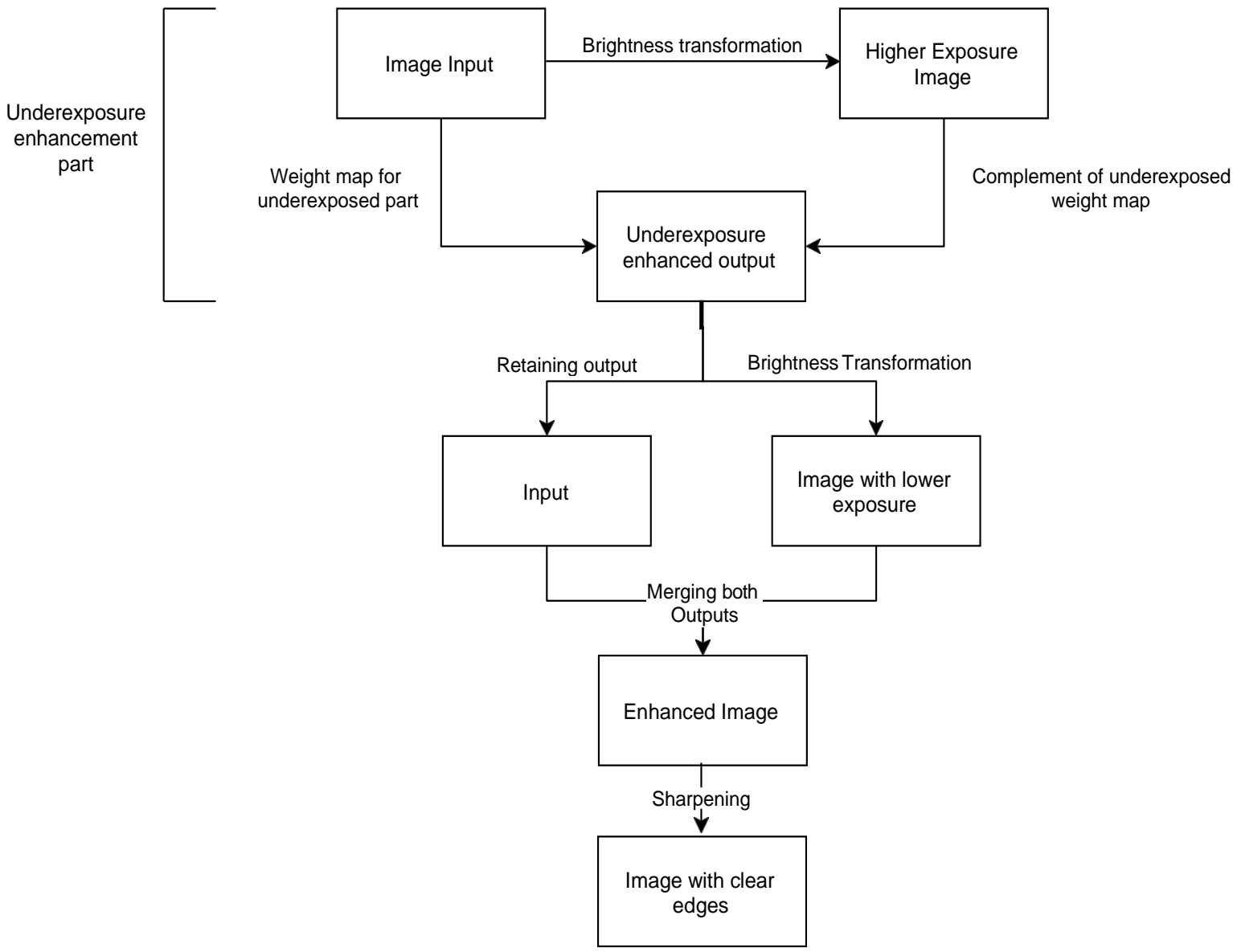

Fig 1: Proposed Flow Diagram

\section{PROPOSED APPROACH}

\subsection{Exposure fusion framework}

The problem is that images with another exposure setting isn't out there fortunately, photos taken with loving are extremely correlated. In the earlier work, this system proposes a camera response model to accurately describe the association between these pictures in order that system generates a series of pictures from the input image [8]. The mapping operates between two pictures that only solely in exposure is called Brightness transform function (BTF) [9]. Given exposure magnitude relation $\mathrm{K}_{\mathrm{i}}$ and $\mathrm{BTF} \mathrm{g}$, system maps the input image $\mathrm{P}$ to the $\mathrm{i}$-th image at intervals the exposure set as

$$
\text { Image }=\mathbf{g}\left(\mathbf{P}, \mathbf{K}_{\mathbf{i}}\right)
$$

In this paper, the algorithm only fuses the input image itself with another exposure to reduce complexity. The fused image is defined as

$$
\text { Output }=W_{0} P^{c}+\left(1-W_{0}\right) g\left(P^{c}, k\right)
$$

\subsection{Weight matrix estimation}

The design of $\mathrm{W}$ is significant to obtaining associate sweetening formula which will enhance the low distinction of under-exposed regions whereas the distinction in wellexposed regions preserved. System need to assign big weight values to well-exposed pixels and small weight values to under-exposed pixels [10]. Intuitively, the burden matrix is absolutely correlative with the scene illumination. Since extremely illuminated regions have huge risk of being wellexposed, they ought to be assign with big weight values to preserve their contrast. The algorithm has a tendency to calculate the weight matrix as:

$$
\text { Weight map }=T_{\mu}
$$

where $T$ is that the scene illumination map and $\mu$ may be a parameter dominant the enhance degree. The estimation of scene illumination map $\mathrm{T}$ is solved by improvement.

\subsection{Exposure Ratio Determination}

In this subdivision, algorithm find the simplest exposure ratio so that the synthetic image is well-exposed within the regions wherever the initial image under-exposed. First, the system excludes the well-exposed pixels and procure a picture that's globally under- exposed [3]. Algorithm have a tendency to merely extract the low illuminated pixels as:

$$
\mathbf{Q}=\{\mathbf{P}(\mathbf{x}) \mid \mathbf{T}(\mathbf{x})<0.5\}
$$

where Q contains only the under-exposed pixels.

The brightness of the images under different exposures changes significantly while the color is basically the same. Therefore, system only consider the brightness component when estimating $\mathrm{k}$. The brightness component $\mathrm{B}$ is defined as the geometric mean of three channel:

$$
B=\sqrt[3]{Q 1} \times \sqrt[3]{Q 2} \times \sqrt[3]{Q 3}
$$

where Q1, Q2 and Q3 are the red, green and blue channel of the input image $\mathrm{Q}$ respectively. 


\section{PROPOSED ALGORITHM}

- Algorithm recommend an image contrast algorithm to produce a certain improvement of contrast.

- Specifically, the weight matrix for image fusion using illumination estimation techniques is designed.

- Then algorithm tend to introduce camera response model to synthesize multi-exposure pictures.

- Next, algorithm tend to find the most effective exposure ratio so the artificial image is well-exposed within the regions wherever the first image under-exposed.

- If the image is overexposed then system is multiplying the overexposed area with the suitable weighing factor.

- Finally, the input image and the artificial image are fused according to the weight matrix to get the improvement result.

- Similarly, the system will perform same algorithm with the appropriate weighing factor for the overexposed white images.

- $\quad$ The proposed algorithm finally performs the sharpening operation to enhance the quality of the output image.

\section{IMPLEMENTATION DETAILS}

In this algorithm, $\mu$ is a parameter that controls the overall degree of enhancement. When $\mu=0$, the resulting $\mathrm{R}$ is equal to $P$, i.e., no enhancement is performed. When $\mu=1$, both the under-exposed pixels and well-exposed pixels are enhanced. When $\mu>1$, pixels may get saturated and the resulting $R$ suff ers from detail loss. so as to perform improvement whereas preserve the well-exposed regions, algorithmic rule set $\mu$ to $1 / 2$ [4].

To maintain the fairness of the comparison, the parameters of the improvement algorithmic rule are all told all told $=$ one, $\mathrm{s}$ $=0.001, \mu=1 / 2$, and also the size of native window $\omega(\mathrm{x})$ is five. The most time-consuming part of this algorithm is illumination map optimization. This system employs the multi-resolution pre- conditioned conjugate gradient solver $((\mathrm{N}))$ to solve it efficiently. so as to additional quickening this formula, formula solve $\mathrm{T}$ mistreatment the down-sampled version of the input image then up-sample the ensuing $\mathrm{T}$ to the initial size. No visual diff erences can be found in the enhanced results if the model down-sample once, but the computational efficiency is greatly improved.

\section{RESULTS}

\subsection{Dark/ Underexposed portion of images}
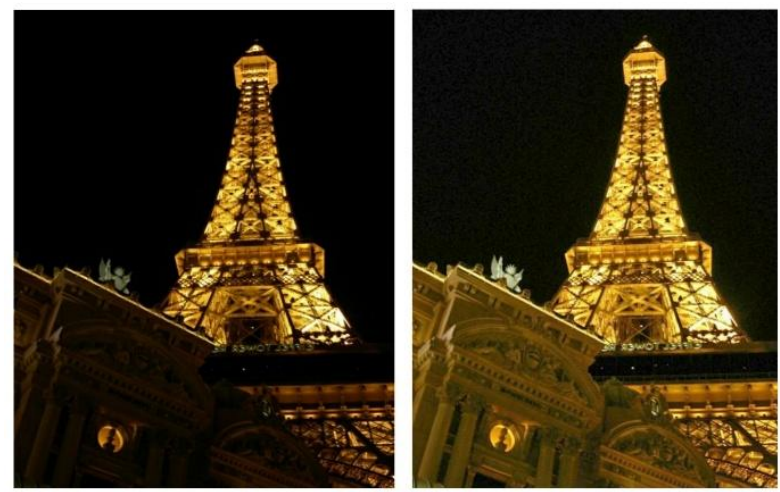

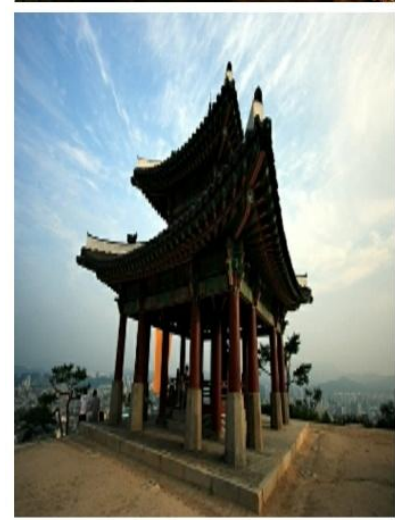

(a)

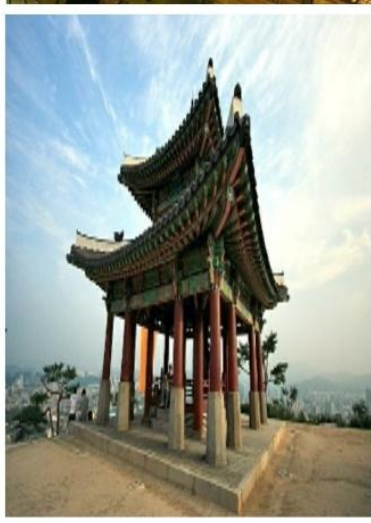

(b)
Fig 2: (a) Input Image (b) Output for underexposed portion of image

\subsection{White/Overexposed portion of images}

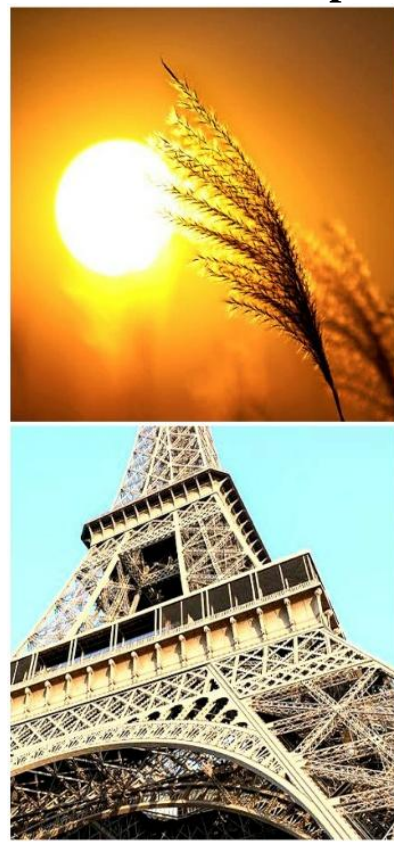

(a)

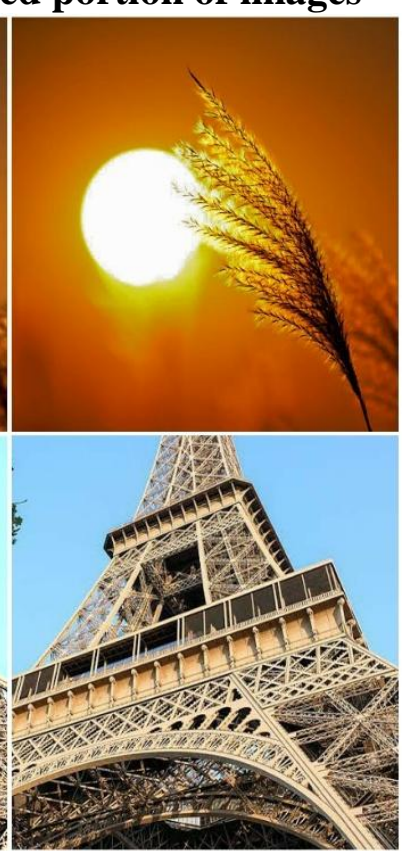

(b)
Fig 3: (a) Input Image (b) Output for overexposed portion of image 


\subsection{Results from proposed system}

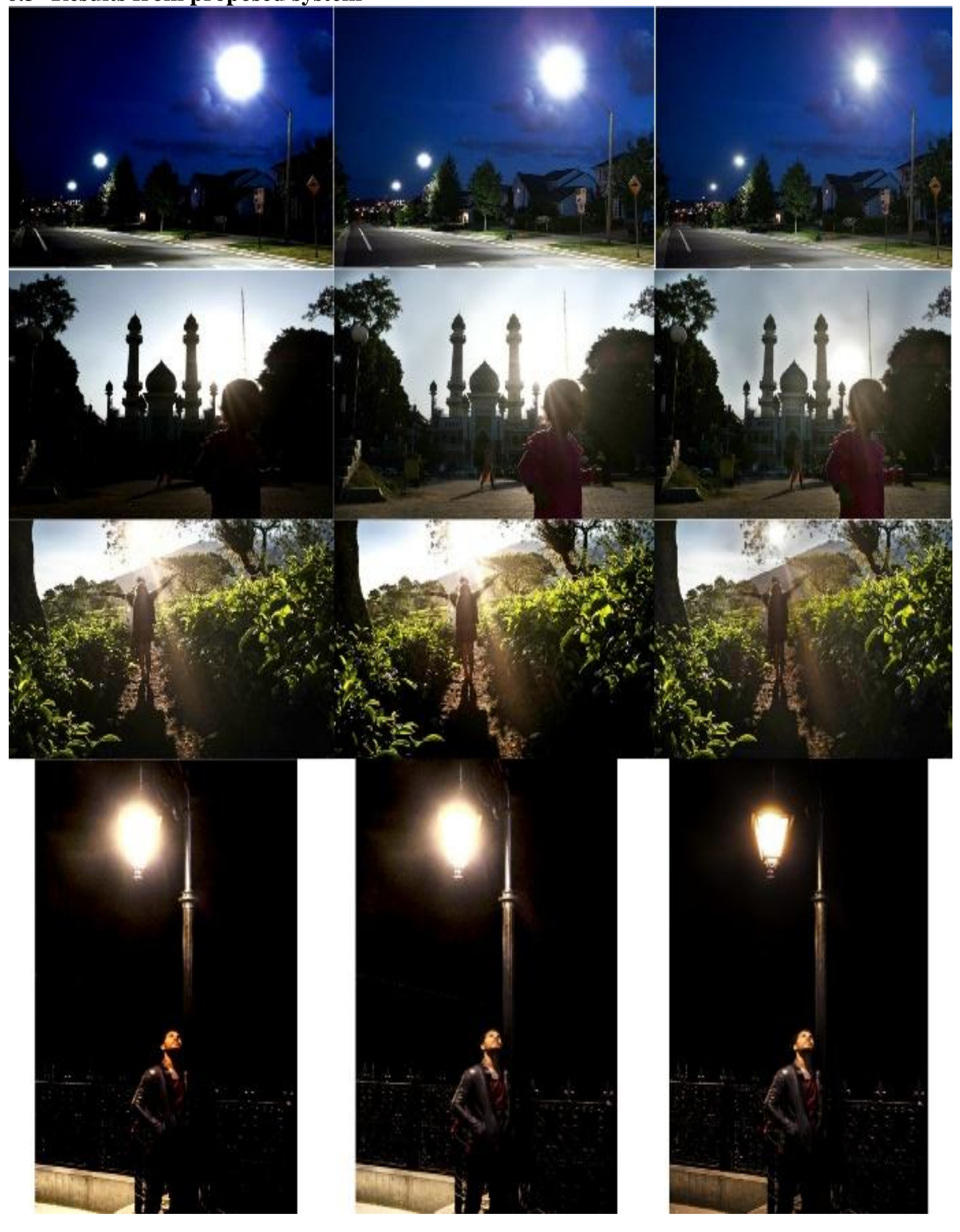

(a) Input

(b) Ying method

(c) Proposed Method

Fig 4: Output of proposed algorithm for images with Underexposed and Overexposed portions followed by sharpening 


\section{RESULT TABLE}

Table 1. Entropy Comparison of results from various methods

\begin{tabular}{|c|l|l|l|l|}
\hline Methods & he & dhe & Ying [4] & $\begin{array}{l}\text { Proposed } \\
\text { System }\end{array}$ \\
\hline Image 1 & 6.0680 & 6.2778 & 6.1298 & 7.5236 \\
\hline Image 2 & 7.1531 & 7.2838 & 7.2084 & 7.4353 \\
\hline Image 3 & 3.2989 & 3.8769 & 3.8730 & 3.5299 \\
\hline Image 4 & 7.2906 & 6.8844 & 7.0059 & 7.0806 \\
\hline
\end{tabular}

Table 2. Objective Evaluation of 6 images processed using he, dhe, ying [4] \& proposed method

\begin{tabular}{|l|l|l|l|l|}
\hline & he & dhe & Ying [4] & $\begin{array}{l}\text { Proposed } \\
\text { Method }\end{array}$ \\
\hline Image 1 & 2.0667 & 2.1333 & 3.0666 & 3.5333 \\
\hline Image 2 & 2.1334 & 2.3334 & 3.1666 & 3.8334 \\
\hline Image 3 & 2.3773 & 2.4000 & 3.1818 & 3.9999 \\
\hline Image 4 & 2.6363 & 2.9090 & 3.2727 & 4.1000 \\
\hline Image 5 & 2.7272 & 3.0000 & 3.5454 & 3.6363 \\
\hline Image 6 & 2.4545 & 2.8181 & 3.0909 & 3.8181 \\
\hline Average & $\mathbf{2 . 3 9 9 2}$ & $\mathbf{2 . 5 9 8 9}$ & $\mathbf{3 . 2 2 0 6}$ & $\mathbf{3 . 8 2 0 1}$ \\
\hline
\end{tabular}

\section{CONCLUSION}

In this paper, the model proposes an exposure fusion framework and an enhancement algorithm to provide contrast enhancement. The advantage of using this model over already existing techniques like histogram equalization and dehazed based techniques is that this model doesn't affect the areas of the input image which are decently exposed and which don't want any further enhancement.

Based on the framework, the model solves 3 problems: (1). The algorithm borrows the illumination estimation techniques to get the weight matrix for image fusion. (2). The algorithm introduces the camera response model to synthesize multiexposure pictures. (3). The model finds the simplest exposure ratio so that the synthetic image is well-exposed within the regions wherever the original input image is under exposed. (4). System reduces the very high brightness or overexposed area by preserving the important features of an input image. The intensity values of saturation are modified keeping hue preserved to preserve the important features.

\section{FUTURE SCOPE}

In many outdoor scenes, cameras cannot make all pixel wellexposed since its dynamic range is limited. Although this system can reveal some under-exposed regions by increasing exposure, the well-exposed regions may become overexposed at the same time. To obtain an image with all pixel well-exposed, system can fuse these images.

Exposure fusion can also create extended depth of field images by fusing together a sequence of images with different depths, comparing the output in images with different depth of field might yield better performance.

\section{REFERENCES}

[1] Beghdadi, A., Le Negrate, A.: Contrast enhancement technique based on local detection of edges. Comput. Vis. Graph. Image Process. 46(2), 162-174 (1989)

[2] Jobson, D.J., Rahman, Z., Woodell, G.A.: A multiscale retinex for bridging the gap between color images and the human observation of scenes. IEEE Trans. Image Process. 6(7), 965-976 (1997)

[3] Lee, C.H., Shih, J.L., Lien, C.C., Han, C.C.: Adaptive multiscale retinex for image contrast enhancement. In: 2013 International Conference on Signal-Image Technology \& Internet-Based Systems

[4] Zhenqiang Y., Ge Li, Yurui R., Ronggang W., and Wenmin W.: A New Image Contrast Enhancement Algorithm Using Exposure Fusion Framework. In: Springer International Publishing AG 2017

[5] Karaduzovic-Hadziabdic, K., Telalovic, J.H., Mantiuk, R.: Subjective and objective evaluation of multi-exposure high dynamic range image deghosting methods (2016)

[6] Ibrahim, H., Kong, N.S.P.: Brightness preserving dynamic histogram equalization for image contrast enhancement. IEEE Trans. Consum. Electron. 53(4), 1752-1758 (2007)

[7] Guo, X.: Lime: a method for low-light image enhancement. In: Proceedings of the 2016 ACM on Multimedia Conference, pp. 87-91. ACM (2016)

[8] Dong, X., Wang, G., Pang, Y., Li, W., Wen, J., Meng, W., Lu, Y.: Fast efficient algorithm for enhancement of low lighting video. In: 2011 IEEE International Conference on Multimedia and Expo, pp. 1-6. IEEE (2011)

[9] Chen, S.D., Ramli, A.R.: Minimum mean brightness error bi-histogram equalization in contrast enhancement. IEEE Trans. Consum. Electron. 49(4), 1310-1319 (2003)

[10] Aydin, T.O., Mantiuk, R., Myszkowski, K., Seidel, H.P.: Dynamic range indepen- dent image quality assessment ACM Trans. Graph. (TOG) 27(3), 69 (2008) 